\title{
Late-Onset Pompe Disease: A Multisystemic Disorder
}

\author{
Mark Roberts* \\ Department of Neurology, Salford Royal NHS Foundation Trust, Manchester, United Kingdom
}

\section{BACKGROUND}

The clinical features of Pompe disease (GSD2) reflect a deficiency of the lysosomal enzyme alpha glucosidase resulting in glycogen accumulation and disruption of myofibrillary architecture in skeletal, respiratory and cardiac muscle. Lysosomal storage disorders produce widespread metabolic dysfunction and involvement of multiple tissues / organs is common.

\section{MATERIALS AND METHODS}

A literature search and analysis of the clinical features of the Salford Cohort of 58 adult-onset patients was conducted to identify additional clinical features.

\section{CONCLUSIONS}

Late-onset Pompe disease is a multisystem disorder, deep phenotyping is recommended to identify the frequency and mechanisms of multi-organ involvement, inform standards of care, and aid recognition of unusual presentations.

\section{RESULTS}

Gastrointestinal problems including dysphagia (5/58, resulting in aspiration in 2), constipation (10/58), poor nutrition (20/58) were common and volvulus occurred in 1 patient. Fatigue and difficulties with concentration were common. Cerebral vascular anomalies were found in 2 of 10 patients screened, with 1 patient presenting with intracranial haemorrhage. Pain was seen frequently, with both musculoskeletal presentations related to scoliosis and lordosis, and myalgia and neuropathic components. Cardiac arrhythmias and valvulopathy were found in a minority of patients $(3,3 / 58)$.

\footnotetext{
*Correspondence to: Mark Roberts, Department of Neurology, Salford Royal NHS Foundation Trust, Manchester M6 8HD, United Kingdom. E-mail: mark.roberts@srft.nhs.uk.
} 